\title{
A Comparative Study of Micro-blog and Micro Message Marketing Mode
}

\author{
Yang Shan \\ School of Economy and Management \\ Shenyang Aerospace University \\ Shenyang, China \\ E-mail: yangshan15927@163.com
}

\author{
Zijian Guo \\ Department of Humanities and Social Sciences \\ Shenyang Aerospace University \\ Shenyang, China \\ E-mail:lelouchsama1@163.com
}

\begin{abstract}
In recent years, the "micro" has become a very popular word. "Micro-blog" and "Micro message", these "micro power" gradually narrowed the distance between people, the global village gradually small, let users enjoy the information age "virtual neighbors" convenience, but also broaden the channels of information dissemination, accelerating the speed of information dissemination. Imperceptibly, changed the way people access information and communicate information. Businessmen will naturally focus to these "micro power" of the marketing ability, a "micro power" bring marketing revolution quietly and health. With the development of mobile Internet and smart phones, micro-marketing underestimated the power of smaller capacity, its low cost, high cost, and integration of various marketing channels to reach the small broad marketing. Micro-marketing, deserved the power of infinity. In this thesis, micro-blog marketing and Micro message marketing as the research object, to explore the application of two kinds of marketing mode of social marketing environment, by analyzing the factors of two kinds of marketing mode of communication, the audience, the scope of the current two marketing patterns and their application.
\end{abstract}

Keywords-social marketing; comparative study; application prospect; micro-blog; micro message

\section{FOREWORD}

With micro-blog, Micro message in marketing to show amazing strength, micro-marketing in this content, socialization, the trend is already moving into a new marketing model, is rapidly shaking up the traditional marketing concepts and models, saying worldwide are setting off a new round of information and communication revolution too.

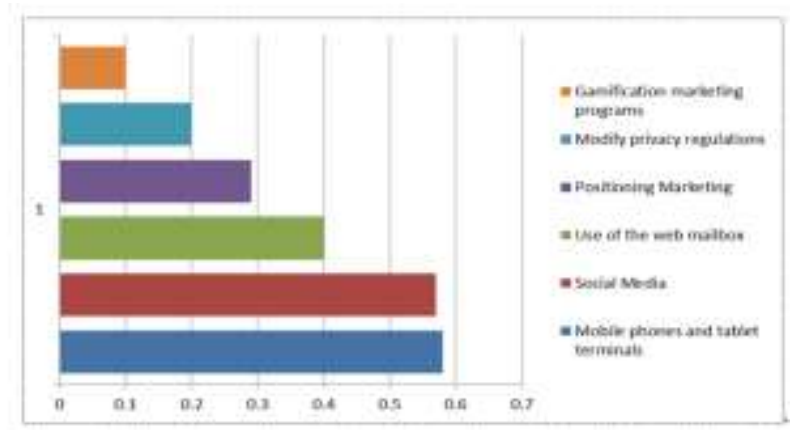

Figure 1. The main factors affecting the network marketing distribution
Traditional advertising and marketing, are the information transfer is the unilateral release of information, and the evolution of the birth of social media micro-blog, letters and other micro-marketing, even more attention to user involvement, emphasis on interaction, reflected in its core values permeate the consumer who "when asked fragmentation" and allow consumers to take the initiative to accept brand messages and interact both.

With the development of mobile Internet and smart phones, micro-marketing "micro" force smaller capacity underestimated, its low cost, high cost, and integration of various marketing channels to reach the small broad marketing. Micro-marketing, deserved the power of infinity.

\section{SOCIAL MEDIA MARKETING MODEL ENVIRONMENT OVERVIEW}

Social media is a two-way exchange of information dissemination based on the shared platform or tool, the difference between social media and traditional online media that social media simulates real-world relationships in the network, and the moon would be a real transmission of information in the Internet sector teams transplant, expanding, making the history of an individual's voice was distant spread.

One way is to use social media marketing social networks, online communities, blog, Wikipedia or other Internet Sites and media to disseminate and publish information, thereby forming marketing, sales, public relations and customer relations services deal with maintenance and pioneering. General social media marketing tools include forums, micro-blog, blog, SNS community, pictures and videos from the media platform or organization through media platform for publishing and dissemination.

Social Media Marketing is committed to long-term relationships with consumers. One by one into the consumer's social network has become one of the nodes, businesses and consumers to establish a "friend" relationship. In this process, businesses and consumers interact; making the company's values, vision services for consumers to understand and accept, effective publicity of enterprises, and consumers can develop a sense of brand awareness, a sense of belonging and improve consumer loyalty. 
Marketing model is a systematic system, not just a marketing tool or a means of marketing methods. Specifically, it includes positioning the enterprise to realize the value of products and services and to determine the scope of the enterprise in the economic position of the ecosystem and the ways and means in order to achieve the stated objectives systematically taken. According to this definition can be drawn, the social networking site's marketing model is based on the sum of the user's attention to the social pooling gain economic and social benefits of a class of ways and means. For social networking sites, it also refers to a communication paradigm for specific user groups and networking products taken.

Compared with the traditional marketing methods, social media marketing campaigns are relatively inexpensive platform. Because it is free or low-cost, anyone can set up a free Twitter account or Facebook page. Enterprises can direct dialogue with consumers, asking and deal with problems. Traditional media adopt a "push" marketing, corporate lack of communication with the user. The use of social networks, an enterprise can implement more personalized, more dynamic marketing. Day after day traffic growth of social networks, the potential of social media marketing cannot be underestimated. However, the use of social media is still a risk that consumers may publish in social media's negative comments about the company. Therefore, before social media marketing, the company should be thinking about how to better use social media.

\section{MICRO-BLOG MARKETING MODEL STUDY}

Micro-blog marketing is a marketing approach through the Micro-blog platform to create value for businesses, individuals and execution. It involves a range including certification, effective fans, topic, blogger, open platform, the overall operation and so on. Micro-blog users do not have to be mandatory to receive certain information, but can "concern" of interest to the person or business information "pull out", you can also keep the "Forward" the message continued to spread, fission. Social media such as the spread of the main advantages and disadvantages businesses, in addition to a free and simple publishing platform, it is more a test of corporate marketing skill. In the context of social media marketing, Twitter marketing is focused on delivering value, layout, accurate positioning interactive content, the system, the development of hot Micro-blog marketing effect also makes it particularly significant.

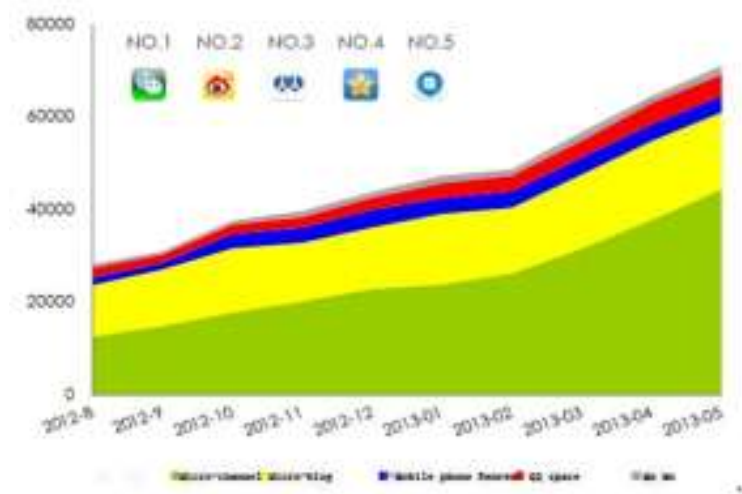

Figure 2. social applications using long TOP5

\section{A. Micro-blog Promotions}

The micro-blog Promotion is an enterprise use Micro-blog released by discounts, promotional offers and other information products. Promotional marketing for the enterprise Micro-blog users concerned about a network marketing model. The use of micro-Bo promotional information, first eliminating the publicity costs, the second largest use of the Micro-blog audience to help their publicity.

\section{B. Micro-blog branding}

Enterprises registered in their account on the Microblog publish their products relevant circumstances establish a corporate image of their brand. Posted on the Micro-blog brand information, answer consumers' questions about products, although more difficult to assess marketing effectiveness, but for businesses. As long as careful management, Micro-blog brand building will be put in the minimum capital, was able to maximize corporate influence and deliver corporate brand connotation propaganda mode.

\section{Micro-blog advertising activities}

Advertising needs to be done taking into account the demands of advertisers, marketing, product value and market demand. More important is "advertised." The era of information explosion, the advertiser's marketing needs have changed. Conventional brand exposure has been unable to meet the expectations of advertisers using online media in-depth marketing advertising is on the rise. Micro-blog use this platform for consumers spiritually uplifting, corporate and brand resonate with consumers emotional advertising that consumers no longer objectionable. Instead, pick on the Micro-blog forward, consumer advertising for businesses to become active person, this ad is more credible, more influential.

\section{RESEARCH ON THE MARKETING MODE OF MICRO-} BLOG

As a Micro message social media in one, it makes people's social from "weak ties" to the phone book for the carrier to "strong relationship." That makes people had Micro message extension of the relationship with the reality of life in the virtual world, making the online world with the real world to achieve a correspondence. It is like an ax as open Micro-blog friends, QQ friends, phone contacts, mail and all other channels, forming a complex social network, along with its endless features such as LBS, 020 two-dimensional code, shake a shake, looking for people who appear near other new features, especially the emergence of a public platform, and more in terms of making the Micro message marketing between strangers or acquaintances have a variety of possible, these features also allow Micro message marketing results more precise and effective

\section{A. Drift bottles - brand activity type, increase brand awareness}

Drift bottles is a simple way to interact with strangers. By "throwing a" voice or text users can publish and put fish in the sea so that other users; through the "pick one," users can get information sent to other users with dialogue. 
B. Location Signature - grassroots advertising style to attract neighboring users

Micro message users can always change their signature, which the enterprise is undoubtedly a convenient free mobile advertising. Micro message user enterprise content can be found near the location of the enterprise, such as user names and signatures through the "View people in the vicinity," the LBS functionbased plug-ins.

\section{The dimensional code of 020 discount style, build a loyal customer base}

The end of 2011, Micro message version 3.5 add new features two-dimensional code, the user can expand the Micro message or friends by scanning the twodimensional code released on other platforms, which is undoubtedly a powerful carrier business promotion marketing. Enterprise set their own brand of twodimensional code and publicly released, and Micro message users can focus on business information by scanning the two-dimensional code, to obtain the corresponding discounts and deals.

\section{The open platform, circle of friends, Micro message public platform - social sharing formula to achieve word of mouth marketing}

Open platform and circle of friends is an effective social platform. By opening the platform and circle of friends, Micro message users can call third-party applications in a session to select and share content to achieve widespread information. The Micro message public platform Micro message on the basis of new functional modules is an open platform and friends circle segments and extending its help enterprises achieve precise information pushed to the target population, in the use of the user's open platform and friends circle, to achieve viral corporate information.

\section{A COMPARATIVE STUDY OF TWO MICRO-BLOG AND MICRO MESSAGE MARKETING MODEL}

\section{A. Features micro-blog marketing model}

\section{1) Low cost.}

Compared with traditional advertising and marketing, micro-blog marketing does not require administrative approval procedures jumbled, but also eliminates the need for companies to pay for the cost of broadcast advertising platform, not only help companies to promote cost savings, but also significant savings in labor costs and time costs. Micro-blog marketing is also more convenient than other network marketing blog marketing and forum marketing, micro-blog. Enterprises can publish any documents related to corporate branding, links, pictures, videos or websites, for free business promotion.

\section{2) High-speed.}

Information dissemination micro-blog geometric let corporate marketing more effective. A micro-Bo has been issued, it can be seen by all concerned enterprise micro-blog users, through interactive forwarding phones and the Internet can be delivered to all potential clients around the world within minutes, maximum short message Receive amount. It can be said micro-blog marketing is the fastest of all marketing tools, one of the most widely spread.

3) Customer-centric.

The use of real-time enterprise micro-blog, smooth communication, feedback function, consumers can quickly and accurately establish close interaction between better to do customer-centric. On the one hand, companies can better communicate their brand philosophy and corporate culture to consumers, to understand the ideas and needs of consumers. On the other hand, you can easily and timely management of the target consumer expectations and cognitive management. Avoiding inappropriate expectations and error estimates. In fact, making the enterprise micro-blog more people have access to the forefront of the market, information is no longer in the layers of the loss and distortion passed, more conducive to business decisionmaking.

\section{B. Features Micro message marketing model}

\section{1) Low marketing costs}

Traditional marketing methods such as television advertisements, newspapers, posters, etc. usually spend a lot of manpower and financial resources, and the Micro message marketing platform based on the Micro message, Micro message functions are available to users free of charge, the process of using only small amounts traffic charges. Compared to traditional marketing methods, Micro message marketing costs extremely low, almost close to free.

2) A large number of potential customers

January 15, 2013, the number of Micro message users has exceeded 300 million, with the advent of micro- era, the number of Micro message users will increase small off, which means a huge marketing. Increasingly expansion of Micro message user groups will become potential customers enterprise Micro message marketing, which has attracted more and more companies join the ranks of Micro message marketing.

3) The precise positioning of marketing

In the Micro message public platform, through one on one attention and push, small businesses can only push relevant information to its products and activities fans can also create their own customer database, so that Micro message becomes effective customer relationship management ((CRM) system enterprise through user groups and geographic control features for the user, the information accurate pushed to the target user.

\section{4) Interactive exchange of information}

Micro message carrier is a smart phone, which means that as long as there is a smart phone at hand, no matter when and where companies can interact with customers to better understand customer needs and then meet customer demand. Although micro-blog marketing can also interact with the fans, but its timeliness is far smaller than the Micro message marketing.

5) The effectiveness of information dissemination

As previously mentioned enterprise is the use of Micro message public platform to push information to the client, in which can guarantee $100 \%$ customer receives a push into the enterprise information. In addition, because the customer is interested in the products or companies voluntarily or two-dimensional 
code scanning business account to add official Micro message input, so when receiving information from the official micro- enterprises believe that they can effectively focus the received information .

\section{6) Diversified marketing model}

Micro message marketing has drift bottles, location signatures, two-dimensional code, development platform, circle of friends, Micro message public platform marketing model, these marketing models have their characteristics small businesses can choose the mode with the same combination for small marketing purposes. In addition, the Micro message can support multiple types of information, can only support a small text, pictures convey, but you can send a voice message, which allows companies to take advantage of Micro message and customer to complete the full range of communication and interaction.

\section{Micro message marketing and marketing micro- blog features comparison}

\section{1) Comparison of characteristics of Micro message and micro-blog}

Micro-blog, including support for sending text, pictures, facial expressions, video, GIF animation work and hypertext links, etc. content. When companies use Micro-blog marketing activities, mainly between marketing objects communicate through text. Microblog can use short text language, pictures, video clips to convey information, but focuses on marketing and marketing marketer interaction between objects, can only rely on at this point Micro-blog hand to carry out the fight between the fans and the text interaction.

Micro message support sending including text, pictures, video, voice, music, animation, facial expressions, etc., as long as can think of, basically Micro message can be sent. For example, as long as the user to send any kind of expression Starbucks China, Starbucks China will use a song "woke up" in the music back to the user, in order to promote their new products. Especially in voice communication is a major highlight of the Micro message software, one voice communication can make communication more cost savings, more convenient, or when users do not bother typing or typing is not convenient, as long as the moving mouths can send information, on the other hand, the same the text consists of a word with a different tone, speaking often produce different meanings, so voice communication to a certain extent also to avoid misunderstandings and ambiguities occur, furthermore what sounds to use as a business user's reply language is also very important, it can give the user a different feel. Voice so that enterprises in the Micro message social media in much closer.

\section{2) Comparison of characteristics of Micro} message and micro-blog media

Micro-blog broke the linear transmission of traditional media and new media is different from the net spread is the spread of a three-dimensional of geometric base fission .It is a truly 4A media ANYTIME, ANYWHERE, ANYONE, ANYTHING than Micro message earlier, Micro-blog appearance will formerly known as " new media" blog, podcast, etc. Push the " old media " in the to subvert the traditional social networking model, through the "concern" and " being concerned ", " forward" function, you can make the news Micro-blog pass 1010 Fax 100 to spread, quick copy, causing concern in the community, has made the spread of maximize the effect .

The essential feature micro-blog propagation mode is "implantable nested". Both functional develop of micro-blog or its information dissemination, or to reconstruct aspects of human social networks, reflected this characteristic. Users concerned about others, while others also are concerned, which is equal to embed circle one after another go, bring a new Micro-blog social networking model. Meanwhile, the micro-blog feature is also embedded in a lot of third-party applications, including the more popular applications are: Micro-blog client, social reading, photo sharing, LBS and socializing.

As a Micro message social media, its inherently has a social function. Introduced the industry's first Micro message LBS positioning social function, opened a new marketing mode location. Precise positioning in favor of the role of LBS enterprises to develop precision marketing, especially for certain sectors when running promotions may be more effective. Micro message 3.0 version pioneered the "Shake " concept, have been demonstrated in a variety of APP, in the quest to strangers on the chain, launched the "Find nearby people " Apply " drift bottles " and other functions , which Dating is also convenient for users to carry out Micro message marketing for the enterprise to provide more and more convenient way, companies can publish a voice or text message, and put into the ocean, drift bottles recovered from users of this information if you are interested in, then it dialogue between business and be able to ; Again looking people in the vicinity, the user can search using the Micro message users nearby , and you can see their signature , if the company marked the business related information in the signature file can be potential marketing object seen signature to become a free gold advertising, these social networking applications revolutionized the way to meet the people 's demand to expand their relationship circle .

Evening August 17, 2012, the Micro message platform was officially opened to the general public users. Through this public platform features interactive marketing style, everyone can use their QQ number to create their own public account, and rely on its implementation with other users on the platform of text, images, voice and video communications and interactive round. With other marketing methods is different, Micro message users can choose whether to accept their decision and passed the public account information.

\section{APPLICATION TREND OF CHINA'S MICRO-BLOG MARKETING AND MICRO MESSAGE MARKETING}

2013, "China's online shopping market transactions amounted to 973.56 billion Yuan, an increase of $27.8 \%$ over 2012, but nearly a third of the proportion of the share compared to western countries, China's online shopping market to be further excavated With a approved business success in marketing micro-blog platform will attract more businesses to try. Accordingly, the development of enterprise micro-blog in quite some 
time, will maintain its high growth trend, which also means that micro-blog marketing industry coverage areas will continue to enrich, industry size will continue to be deepened and expanded.

\section{A. The trend of application of micro-blog marketing}

1) "The big corporate public relations, different development of small enterprise sales"

From the number of terms, at present in the microblog platform marketing has effective enterprise or minority. Micro-blog for most enterprises is an official news platform. After all micro-blog economy has just emerged, even if the enterprise has been aware of the need to change the strategic direction, the short term can't change the dependence on traditional marketing methods and the relationship with existing customer, need time cost. On the other hand, microblog function more perfect, more perfect service, in the "infrastructure" equal condition ,micro-blog Marketing ideas has become the core competitiveness of enterprises. In contrast, large enterprises have more manpower advantage, more important microblog in brand image and corporate public relations in terms of value, its development will become the integration of social marketing platform. More small and medium enterprises is through the micro-blog product sales in the United States there have been a number of live micro-blog rely on product sales company.

2) micro-blog marketing means to diversified

For the current enterprise micro-blog application is still in its infancy, the use of the function, but the majority of published content, comments and forward user information. Interaction also "concerned about the activities of ten prizes ten + Review ten forward" based. Micro-blog marketing in many respects still need to improve and enhance the Chinese: the understanding of the micro-blog to be improved, the registration and use can be more convenient and professional segments require more intensive, information classification is not perfect, false information management and control. At the same time companies need to think, how to shortterm activities micro-blog marketing, branding becomes persistent. Worthy of recognition, the enterprise microblog and micro-blog marketing awareness level, must practice with the depth continues to increase. Word of mouth marketing, relationship marketing, event marketing, social network marketing tools, combined with the characteristics micro-blog platform will bring more "surprise" companies. Micro-blog in the future, as a collection of real-time search, data analysis, based location services in a marketing tool, will have a greater role.

\section{3) Micro-blog marketing localization services}

User demand for localization services is a practical nature, micro-blog features just fit a certain extent this point. Data show has opened micro-blog enterprises are mainly distributed in 22 food and beverage industry enterprises in which nearly 50,000 the number of active fans enterprise micro-blog champion scale and proportion interactive fans are in a leading position. In addition to food and beverage class tourist hotel, entertainment and shopping malls are enterprise microblog concern. Dining, entertainment, shopping is the user's life with the user's basic consumer lives so users are more likely to localization services enterprise microblog generate feedback to facilitate the sharing of information conducive to enterprise branding and establish a reputation.

\section{B. Application of Micro message marketing trends}

1) adding search capabilities to achieve precision marketing

4C marketing concept is the core point of view to provide products based on user needs, once the user has certain needs, open Micro message search engine, the search advertising businesses to provide information that can make the company's products reach the right target customer information to achieve precision marketing, this active non-intrusive marketing will be the mainstream Micro message marketing, and traditional marketing content may lead to user dissatisfaction, even uninstall Micro message applications.

2) improve Micro message CRM, focusing on maintaining customer loyalty

CRM Customer relationship management, Micro message must have a dedicated customer service department or equity security department, Micro message privacy characteristics determine the need to ensure that Micro message users' privacy, mobile CRM must protect the Micro message user queries promptly resolved, especially for Micro message users complaints, most of the Micro message customer account and can't act as the role of Micro message marketing companies must have a separate customer service department in order to prevent the number of fans surged by surprise. Note that the process of maintaining customer loyalty, loyal customer base word of mouth marketing can often bring excellent results.

3) Micro message marketing to overcome the current problems

Security issues Micro message. As the Micro message is now in its infancy, people in the network community are diverse, there are some security issues, and Micro message no safety supervision center, there is a security vulnerability in many areas, due to the Micro message marketing does not have a secure payment platform and legal safeguards system, the existence of transactions in Micro message process certain risks and safety hazards, so in order to ensure the security of transactions, should be introduced in collaboration with third-party payment or bank, etc. such as the introduction of Caifutong, confirm the safety of the transaction environment.

The authenticity of the information can't produce Micro message identification, can't determine the validity of the information, thus causing the user may be deceived, Chong Micro message is no clear information monitoring center, resulting in uncertainty and false information, easy to arouse suspicion user and questioned, so it should set up appropriate information security center to monitor the safety and authenticity of the information.

Micro message marketing subject to geographical restrictions, not well widespread, due to the Micro message is spread primarily by means of Micro message positioning system, which limits the regional Micro 
message marketing, so in order to better serve customers, businesses can develop the appropriate version and marketing business version, so you can better and more accurate marketing.

The fast pace of development of Micro message, the software itself is also flawed, such as adding a friend and more will appear card phenomenon in Saipan system, so the Micro message marketing, e-commerce has had an impact, missed some customer groups and Micro message itself is there are many platforms when it will fail, so the foundation is not stable implementation, conduct e-commerce marketing from great suffering, so it should be optimized software , user groups, to create a better and more accurate software and features.

\section{REFERENCES}

[1] Qiu Shi Yu Wu Si Mao Xiuli, micro-blog and micro-channel marketing capabilities compared with the expected future, "China Business \& Trade", 2013 (7)

[2] Long Jue Yu Wang Mingyu, micro-channel marketing wave struck, micro-blog marketing go, "China Business \& Trade", 2013 (11)
[3] Wu Shuang , micro-blog marketing out of "Northeast Window", 2013

[4] Huazhu white, from a social marketing perspective, microchannel is also no substitute micro-blog, "Modern Enterprise Education" , 2013

[5] Xu Jin, micro-blog micro-channel open era, China Direct, July 2012

[6] Shiya Guang "enterprise micro-blog marketing strategy research", East China Normal University, 2011

[7] Zhang $\mathrm{Xu} \mathrm{Er}$, micro-channel to promote viral marketing analysis, news dissemination, 2012 (6)

[8] Zhang Xu Seoul. Micro-channel to promote viral marketing analysis of Journalism and Communication, 2012 (6)

[9] Qin Kai. Pros and cons of micro-channel enterprise marketing, e-commerce, 2012 (11)

[10] Lu Jing rain micro letter, put into reality Precision Marketing, Sales and Marketing (Management Edition), 2012 (11)

[11] Wang Shanshan, compared micro-blog micro-channel marketing capabilities and analysis, Chinese e-commerce, 2013 (23)

[12] Wang Shijie, thinking about the micro-channel marketing status and countermeasures, information and computers, 2013 (1) 